\title{
Motivators of Software Process Improvement: An Analysis of Vietnamese Practitioners' Views
}

\author{
Mahmood Niazi ${ }^{1}$, Muhammad Ali Babar ${ }^{2}$ \\ ${ }^{1}$ School of Computing and Mathematics, Keele University, UK, ${ }^{2}$ Lero, University of Limerick, Ireland \\ ${ }^{1}$ mkniazi@cs.keele.ac.uk, ${ }^{2}$ muhammad.alibabar@ul.ie
}

\begin{abstract}
OBJECTIVE - In this paper we present findings from an empirical study that was aimed at determining the software process improvement (SPI) motivators. The main objective of this study is to provide SPI practitioners with some insight into designing appropriate SPI implementation strategies and to maximize practitioner support for SPI.

METHOD - We used face-to-face questionnaire based survey sessions as our main approach to collect data from twenty-three software development practitioners of eight Vietnamese software development companies. We asked practitioners to choose and rank various SPI motivators against the five types of assessments (high, medium, low, zero, or do not know). From this, we propose the notion of 'perceived value' associated with each SPI motivator.

RESULTS - We have identified 6 'high' perceived value SPI motivators that are generally considered critical for successfully implementing SPI initiatives. These motivators are: cost beneficial, job satisfaction, knowledgeable team leaders, maintainable/easy processes, shared best practices, and top-down commitment. Our results show that developers are highly motivated by: career prospects, communication, cost beneficial, empowerment, knowledgeable team leaders, maintainable/easy processes, resources, shared best practices, top-down commitment, and visible success; Managers are motivated by: job satisfaction, knowledgeable team leaders, maintainable/easy processes, meeting targets, shared best practices, and top-down commitment. Our results also show that practitioners of small and medium sized companies are highly motivated by: cost beneficial, job satisfaction, knowledgeable team leaders, and maintainable/easy processes; practitioners of large companies are highly motivated by: cost beneficial, reward schemes, shared best practices and top-down commitment.

CONCLUSIONS - We believe practitioners use SPI motivators on the basis of the perceived value of that motivator. This focus on perceived value associated with each SPI motivator, offers SPI practitioners opportunities for implementing motivators to improve organisation's SPI implementation capabilities. By analysing the degree to which practitioners use each of the SPI motivators, we can identify a number of critical SPI motivators for SPI practitioners.
\end{abstract}

Keywords: Software Process Improvement, Motivators, Empirical Study.

\section{INTRODUCTION}

To improve software processes, many software process improvement (SPI) models and standards have been developed (ISO/IEC-15504, 1998; SEI, 2002). In order to successfully implement SPI standards and models, we need to be constantly aware of what really motivates practitioners in real life and to understand what SPI motivators are perceived to be useful by practitioners. This will enable us to position our research within an appropriate context (Davis and Hickey, 2002). Moreover, it is also important for people who manage SPI in companies to discover which motivators will highly motivate software practitioners in successfully implementing SPI standards and models, as research shows that the effort put into SPI models and standards can assist in producing high quality software, reducing cost and time, and increasing productivity (Butler, 1995; Pitterman, 2000; Yamamura, 1999). It is also suggested that SPI has a higher chance of success in companies where practitioners are highly motivated for supporting SPI (Baddoo and Hall, 2002). The knowledge of employee's SPI motivation is expected to help develop new or improved SPI approaches, whose adoption will better match companies' objectives, and should also help SPI managers to design effective implementation strategies for SPI programmes in their companies.

Previously, other researchers (Baddoo and Hall, 2002; Khalil et al, 1997) have also conducted studies to identify motivators of software development practitioners. Our research is aimed at not only extending the findings of those studies by conducting a similar study in a different culture; but we also intend to expand this type of research by 
understanding the relative value of each identified motivator perceived by practitioners. We believe that software practitioners may associate different value to different SPI motivators. Moreover, it is also possible that SPI motivators may vary from one geographical region to another. As part of a large project about the SPI motivation, Keele University and National ICT Australia has been carrying out a research project to investigate the SPI motivators in the Asia-pacific region. The results of this project are expected not only help software practitioners to understand the usage of SPI motivators in the Asia-pacific region but also help them to compare SPI motivators identified in other regions (Baddoo and Hall, 2002; Khalil et al, 1997).

The paper reports the findings of one part of our research project aimed at identifying the factors that are perceived by Vietnamese software practitioners as SPI motivators to support SPI initiatives. The findings of this research combined with the findings of the previous similar studies can shed some light on the motivators that should be considered critical when designing SPI implementation strategies.

There are two research questions that have motivated the work reported in this paper:

RQ1. What would motivate practitioners to support a SPI initiative?

RQ2. What SPI motivators have the highest perceived value for practitioners?

This paper is organised as follows. Section 2 describes background and motivation. Section 3 describes the concept of perceived value. Section 4 describes the research design. In Section 5 findings are presented and analysed. Section 6 provides the discussion and conclusion.

\section{BACKGROUND AND MOTIVATION}

In the last few years we have seen technical quality initiatives, such as CASE tools, and organisational initiatives, such as CMM (Paulk et al, 1993), and more recently CMMI (SEI, 2002) which is in order to improve software processes. It has been suggested that whether a quality initiative is technical or organisational, ineffective implementation of these initiatives can significantly affect the success of SPI efforts (Florence, 2001; Niazi et al, 2005b; Niazi et al, 2006). McDermid and Bennet have argued that the human factors to SPI have been ignored and this has damaged the effectiveness of SPI programmes (McDermid and Bennet, 1999). Hall and Wilson have also suggested that the experiences, opinions, and perceptions of software practitioners impact indirectly on the quality of software produced (Hall and Wilson, 1997; Hall and Wilson, 1998). Several other studies have reported that people factors influence the outcomes of the SPI activities. For example, Wilson et al. emphasized the importance of people factors in SPI success (Wilson et al, 2001). Herbsleb and Goldenson reported a relationship between high process maturity and high staff morale (Herbsleb and Goldenson, 1996). Kaltio and Kinnula have also reported that people factors were most important in deploying defined software processes (Kaltio and Kinnula, 2000). These findings highlight the importance of managers' understanding of the factors that influence software practitioners' behaviour towards SPI initiatives.

Baddoo and Hall reported 30 SPI motivators based on 49 focus group discussions in 13 UK software companies (Baddoo and Hall, 2002). Their findings were aimed at helping managers to design appropriate SPI implementation strategies that have wide support from practitioners. They found that most motivators were specific to a particular practitioner group with only a few common to all practitioner groups. Prior to Baddoo and Hall, Khalil et al. reported a comparative analysis of the motivators of Egyptian and Western software engineers (Khalil et al, 1997). Like other studies (e.g., (Sharp et al, 1999; Warden and Nicholson, 1995)), Khalil et al. concluded that software practitioners, as a group, possess unique characteristics, which should be taken into account while designing work related strategies for them. Their study also found that software practitioners usually have a low need of social interaction and experience lack of job autonomy and feedback from their supervisors. Similar findings have been reported by Warden and Nicholson (1995) based on a Job Diagnostics Questionnaire (Hackman and Oldman, 1976) administered to a group of software quality practitioners.

Apart from extending the above-mentioned studies conducted for understanding the practitioners' motivation for SPI, our research also identifies the perceived value of each identified SPI motivator. Moreover, our study has been conducted in a country that is increasing becoming an attractive destination for software development outsourcing from Western and Asian countries alike (Chidamber, 2003). That is why we believe that there is a need of shedding some light on the factors that motivate Vietnamese practitioners to support SPI initiatives.

\section{PERCEIVED VALUE}

In this particular study we define 'perceived value' to mean the degree of importance placed on a SPI motivator by practitioners. This may be considered as a subjective view since it is provided by the participants of this study. However, the participants of this study were considered knowledgeable about the SPI approaches and were actively 
participating in the SPI initiatives of their respective companies. That is why we assume that their opinion is grounded in in-depth knowledge and significant experience of the factors that influence the SPI efforts in industrial contexts.

In order to describe the notion of perceived value within SPI motivators, it is important to decide on the "criticality" of a perceived value. For this purpose, we have used the following criterion:

- If the majority of respondents $(\geq 50 \%)$ thought that a motivator had high value then we treat that motivator as critical.

A similar approach has been used in the literature (Niazi et al, 2005b; Rainer and Hall, 2002b). The perceived values of SPI motivators can act as a guide for SPI practitioners when implementing SPI initiatives.

\section{RESEARCH DESIGN}

\subsection{Study Methodology}

We used face-to-face questionnaire based survey sessions as our main approach to collect data from twenty-three software development practitioners of eight Vietnamese software development companies, which had initiated SPI programs. Although we do not claim this is a statistically representative sample, Table 1 does show that the participants of this study were working for companies of varying sizes. It is further worth mentioning that the data was collected from practitioners who were involved in tackling real SPI implementation issues on a daily basis in their respective companies. Therefore we have high confidence in the accuracy of their responses about their personal motivators of SPI.

It is further important to acknowledge that the practitioners sampled within companies are representative of practitioners in organisations as a whole. A truly representative sample is impossible to attain and the researcher should try to remove as much of the sample bias as possible (Coolican, 1999). In order to make the sample fairly representative of SPI practitioners in particular company, different groups of practitioners from each company were selected to participate in this research. The sample of practitioners involved in this research includes developers, quality analysts, SQA team leaders, SQA managers, project managers, and senior management. Thus the sample is not random but a convenience sample, because we sought a response from a person with a specific role within a software development company. The practitioners who participated in this study fall into two main categories:

- "Developers" consisting of programmer/ analyst/ SQA coordinator.

- "Managers" consisting of team leader/ project manager, and senior managers.

We used a closed ended questionnaire as an instrument to collect self-reported data. Our questionnaire was based on the SPI motivators reported by Baddoo and Hall (2002) and Khalil et al. (1997). The definition of motivators is provided in appendix A (Baddoo and Hall, 2002). The questionnaire was also designed to elicit the importance that each respondent places on each identified motivator (perceived value). In order to describe the importance of motivators, the respondents were supposed to mention each identified motivator's relative value (i.e., High value, Medium value, Low value, Zero value, or Not sure).

In order to analyse the perceived value of each identified SPI motivator, the occurrence of a perceived value (high, medium, low, zero) in each questionnaire was counted. By comparing the occurrences of one SPI motivator's perceived values obtained against the occurrences of other SPI motivators' perceived value, the relative importance of each motivator has been identified. We have also used this approach to identifying highly and lowly valued requirements engineering practices reported in (Niazi et al, 2005a).

\subsection{Study Procedure}

The responses to the questionnaire were gathered during September 2005. The respondents were employees of eight software development companies, which had agreed to participate in our research. Two of the companies sent apology before the data collection process started as their employees were too busy to be able to participate in the study. We had requested the participating companies to nominate at least two employees to participate in this study. We managed to administer the survey instrument to more than two software practitioners from some companies. Although all the participants were well-versed in English and the questionnaire was in English. Moreover, the research team had a Vietnamese speaking researcher, who could have provided necessary explanation if required.

Two weeks before the execution of the study procedure the participants were provided, via email, the details of the process and procedures for the survey sessions. In order to safeguard the confidentiality of the data and privacy of the participants, we also sent a statement of the ethical principles the research team would follow. However, we did not 
provide the participants with the questionnaire in advance in order to avoid any pre-judgment bias. At the start of each survey session, the participants were again assured that their data would not be accessible to anyone except the research team. Furthermore, we explicitly made it clear to the participants and their companies that the research team would not share the data with anyone in a way that could reveal any participant's or company's identity. The researchers also explained to the participants the meanings of motivators for SPI. It was also explained to them that they were supposed to identify their personal motivators for SPI efforts. Furthermore, they were briefed about the need and importance of mentioning the respective importance of each identified motivator. All the survey sessions were conducted in face-to-face meetings at the participants' offices in an environment free of distractions. Additionally, the participants were also asked if they could have thought of SPI motivators others than those included in the questionnaire.

TABLE 1: DEMOGRAPHICS OF THE PARTICIPANTS' COMPANIES

\begin{tabular}{|l|c|c|l|}
\hline Company Id & $\begin{array}{c}\text { Company size } \\
\text { (Number of } \\
\text { employees) }\end{array}$ & $\begin{array}{c}\text { Number of } \\
\text { participants }\end{array}$ & \multicolumn{1}{|c|}{ Titles of participants } \\
\hline C1 & 80 & 2 & Project manager, Team leader \\
\hline C2 & 70 & 6 & $\begin{array}{l}\text { Developer, Test leader, Programmer, Divisional head, } \\
\text { Developer, QA manager }\end{array}$ \\
\hline C3 & 150 & 2 & Chief Technology Officer, QA manager \\
\hline C4 & 150 & 3 & Design team leader, R\&D team leader, QA team leader \\
\hline C5 & 700 & 2 & Project Manager, Process quality manager \\
\hline C6 & 150 & 2 & QA Manager, Operation manager \\
\hline C7 & 50 & 4 & QA manager, Project engineer, Project leader, Project leader \\
\hline C8 & 200 & 2 & QA coordinator, QA manager \\
\hline
\end{tabular}

\section{RESULTS}

\subsection{Demographics}

Table 1 shows the profile of the participants. Twenty-three surveys were conducted in eight companies with programmer/ analyst/ SQA coordinator, team leader/ project manager, and senior managers/ directors. We also wanted to analyse the responses based on a respondent's company size. To achieve this objective, we decided to cluster the participating companies into different groups based on their sizes in terms of number of software development staff. Using the company size definition provided by the Australian Bureau of Statistics (Trewin and D, 2002), we divided these companies into three categories: SMALL (0 to => 19 employees), MEDIUM (20 to => 199 employees), and LARGE (200+ employees). According to this categorization of the companies, six are small-mediumsized and two are large-sized companies.

\subsection{SPI motivators identified by all practitioners}

Table 2 shows that the most common 'high' value motivator perceived by the respondents is 'maintainable/easy processes' (15 out of 23). One reason for this can be the extra time and effort that practitioners are expected to allocate to different activities related to SPI initiatives (Baddoo et al, 2000). The extra time and effort are required because software practitioners have to carry out SPI activities in addition to their routine software development activities (Niazi et al, 2004). 'Top-down commitment' is also another most common 'high' perceived value SPI motivator. This finding supports the results of several studies that emphasised the importance of top-down commitment/ higher management support for successful implementation of SPI programmes (Basili et al, 2002; Butler, 1997; Goldenson and Herbsleb, 1995; Niazi et al, 2006; Pitterman, 2000).

More than half of practitioners consider 'cost beneficial' as an important SPI motivator. This is quite understandable as research shows that the effort put into SPI can assist in producing high quality software, reducing cost and time, and increasing productivity and employee satisfaction (Ashrafi, 2003; Butler, 1995; Jiang et al, 2004; Pitterman, 2000; Yamamura, 1999). We have found that 'knowledgeable team leaders' is also a common 'high' value motivator for SPI. 
We argue that knowledgeable and experienced staff should be involved in SPI initiative because they have detailed knowledge and experience of SPI implementation. With experienced staff less rework of the documentation items is required, issues can be resolved quickly, and chances of destruction are reduced (Kautz and Nielsen, 2000; Moitra, 1998). Different researchers have discussed the importance of having knowledgeable and experienced staff for a successful SPI implementation. For example, Kautz and Nielsen (2000) describe that SPI implementation in one company failed because "the staff and technical director had no prior experience with SPI and its potential benefits" (Kautz and Nielsen, 2000:pp4); Moitra (1998) provides a good account of the problems and difficulties of change management aspect of SPI implementation and identifies inexperienced staff as one of the barriers to a successful SPI implementation. Emphasising the need of having people with SPI experience on the SPI team, he says "the quality and process improvement people are often quite theoretical - they themselves do not understand quite well the existing software development processes and the context in which they are used" (Moitra, 1998:pp202).

TABLE 2: SPI MOTIVATORS IDENTIFIED BY ALL PRACTITIONERS

\begin{tabular}{|c|c|c|c|c|c|c|}
\hline \multirow[t]{2}{*}{ SPI motivators } & \multicolumn{6}{|c|}{$\begin{array}{c}\text { Occurrence in survey }(n=23) \\
\text { Perceived value }\end{array}$} \\
\hline & High & Medium & Low & Zero & Not sure & No response \\
\hline Automation & 5 & 11 & 2 & 2 & 2 & 1 \\
\hline Autonomy & 6 & 7 & 2 & 3 & 5 & 0 \\
\hline Bottom-up initiatives & 5 & 9 & 3 & 0 & 5 & 1 \\
\hline Career prospects & 8 & 9 & 4 & 0 & 1 & 1 \\
\hline Communication & 7 & 13 & 2 & 0 & 0 & 1 \\
\hline Compulsory & 2 & 9 & 7 & 1 & 3 & 1 \\
\hline Cost beneficial & 12 & 6 & 4 & 0 & 0 & 1 \\
\hline Critical mass & 0 & 8 & 5 & 2 & 4 & 4 \\
\hline Eliminates bureaucracy & 6 & 10 & 5 & 0 & 1 & 1 \\
\hline Empowerment & 5 & 11 & 4 & 1 & 2 & 0 \\
\hline External audits & 5 & 12 & 3 & 0 & 3 & 0 \\
\hline Feedback & 9 & 12 & 2 & 0 & 0 & 0 \\
\hline Job satisfaction & 11 & 10 & 0 & 0 & 2 & 0 \\
\hline Justifiable benefits & 5 & 15 & 2 & 0 & 0 & 1 \\
\hline Knowledgeable team leaders & 12 & 10 & 1 & 0 & 0 & 0 \\
\hline Maintainable/easy processes & 15 & 7 & 0 & 0 & 0 & 1 \\
\hline Meeting targets & 9 & 10 & 0 & 1 & 2 & 1 \\
\hline Phased introduction & 1 & 7 & 5 & 1 & 6 & 3 \\
\hline Process ownership & 4 & 13 & 3 & 1 & 2 & 0 \\
\hline Reduced admin & 2 & 12 & 5 & 0 & 3 & 1 \\
\hline Resources & 7 & 12 & 3 & 0 & 1 & 0 \\
\hline Reward schemes & 6 & 5 & 8 & 0 & 2 & 2 \\
\hline Saleability & 4 & 9 & 4 & 0 & 5 & 1 \\
\hline Shared best practices & 11 & 9 & 1 & 0 & 1 & 1 \\
\hline SPI forum & 4 & 7 & 3 & 3 & 4 & 2 \\
\hline Standardisation & 9 & 10 & 2 & 0 & 2 & 0 \\
\hline Taller hierarchy & 5 & 8 & 5 & 1 & 4 & 0 \\
\hline Task forces & 9 & 8 & 5 & 0 & 0 & 1 \\
\hline Top-down commitment & 13 & 6 & 2 & 0 & 1 & 1 \\
\hline Training & 7 & 16 & 0 & 0 & 0 & 0 \\
\hline Visible success & 10 & 11 & 1 & 0 & 1 & 0 \\
\hline
\end{tabular}

'Training' is considered as the most common 'medium' value SPI motivator (16 out of 23). Other most common 'medium' perceived value motivators are 'communication', 'process ownership', 'external audits', 'feedback', 'resources' and 'reduced admin'. It is quite interesting to find that 'resources' is perceived to be a medium value motivator as many studies have reported lack of sufficient resources as one of the major reasons for SPI failures (Florence, 2001; Kautz and Nielsen, 2000). For example, Florence (2001) found that MITRE Corporation's attempt to achieve CMMI level 4 failed 
because management did not allocate sufficient resources; however, the company ended up achieving CMMI level 3 certification as the allocated resources enabled the company to be at that level only. Kautz and Nielsen (2000) report that SPI initiative failed in one company because project managers were reluctant to allocate resources from their projects to SPI activities; however, SPI was successfully implemented in another company, where project managers fully supported SPI initiatives (Kautz and Nielsen, 2000). It has also been found that management often embark on SPI initiatives without having a sufficient knowledge of the resources required for a SPI programme. In some organisations, management assumes that SPI can successfully implemented with a very little resources, while in other organisation management may not consider a SPI programme as a real project (Niazi et al, 2004).

'Reward Schemes' is a 'low' perceived value motivator. Some organisations make SPI compulsory for their staff members. We have found that SPI 'compulsory' as a 'low' perceived value motivator. This has also confirmed our previous findings (Niazi et al, 2004) in which we have identified a 'time pressure' as a barrier to SPI implementation. Normally SPI is made compulsory in organisations and software practitioners are expected to do SPI implementation in addition to their day-to-day software development activities (Niazi et al, 2004).

\subsection{SPI motivators identified by different group of practitioners}

Table 3 shows the results of the analysis of the responses based on the participants' roles in their companies. Table 3 also shows the relative value of the each SPI motivator perceived by both groups of the respondents. We believe that if a SPI motivator is perceived to have high impact on gaining support for SPI initiative by practitioners of both groups, that SPI motivator should be considered critically important in designing SPI strategies. This is because we have a motivator that is replicated across all groups of practitioners.

It is obvious from Table 3 that 'cost beneficial' is the most common 'high value' motivator perceived by the developers (6 out of 8). Other most common 'high value' motivators perceived by the developers group are: top-down commitment and visible success. Our results have confirmed that developers need strong management support for SPI activities (Goldenson and Herbsleb, 1995; Niazi et al, 2006). Developers also need visible success for SPI initiatives. SPI is a long-term approach and it takes time to realise the real benefits of this approach (SEI, 2004). Our results also show that developers are highly motivated by: career prospects, communication, empowerment, knowledgeable team leaders, maintainable/easy processes, resources, and shared best practices. The most common 'medium value' motivators perceived by developers are: automation, external audits and training.

Table 3 shows that more than $70 \%$ of the managers have mentioned 'Maintainable/easy processes' as a 'high' perceived motivator. Little attention has been paid to the effective implementation of SPI initiatives, which has also been found in other studies (Goldenson and Herbsleb, 1995; Niazi, 2006). Other studies also show that $67 \%$ of the SPI managers want guidance on how to implement and maintain SPI activities (Herbsleb and Goldenson, 1996). This motivator suggests that in the opinion of managers a maintainable and easy SPI process can play a vital role in the implementation of SPI programmes. Other 'high' perceived value motivators cited by the managers are: Knowledgeable team leaders, job satisfaction, meeting targets, shared best practices, and top-down commitment. The most common 'medium value' motivators perceived by managers are (10 out of 15): communication and training.

\subsection{SPI motivators based on respondents' company sizes}

According to the company size categorisation provided in section 5.1 , nineteen participants were working for smallmedium size companies; while only four participants were working for large size companies. Our analysis of the SPI motivators perceived by practitioners of different sizes of companies revealed some interesting insights. It was found that a majority of the respondents from small-medium size companies cited 'maintainable/easy processes' and 'knowledgeable team leaders' as the 'high' perceived value motivators. Moreover, nearly $50 \%$ of them also reported 'cost beneficial' and 'job satisfaction' as 'high' perceived motivators. More than $70 \%$ of small-medium size companies' employees perceived 'training' as a 'medium' value motivator.

Our results confirm previous findings in which training is described as an important factor for SPI: Fitzgerald and O'Kane (1999) have identified good training as one of the critical factors and stated that Motorola considers employee training as critical and the training department of Motorola develops the annual training plans for all the employees. Fowler et al (1999) also describe the lessons learned for SPI at Xerox and state that training for all levels of practitioners was critical to SPI success. In another study, Rainer and Hall (2002a) have also identified 'training and mentoring' as most important factor in SPI. The authors state that 'training and mentoring' is identified as the most prominent factor not only because it is recognised by the most number of samples, but also because it is recognised by two sets of companies with successful SPI (Rainer and Hall, 2002a). Other 'medium' perceived value motivators cited by 
our study's respondents coming from small-medium size companies are: Justifiable benefits, communications, and feedback.

TABLE 3: SPI MOTIVATORS IDENTIFIED BY DIFFERENT GROUP OF PRACTITIONERS

\begin{tabular}{|c|c|c|c|c|c|c|c|c|c|c|}
\hline \multirow[t]{2}{*}{ SPI motivators } & \multicolumn{5}{|c|}{ Developer $(n=8)$} & \multicolumn{5}{|c|}{ Manager $(n=15)$} \\
\hline & High & Medium & Low & Zero & $\begin{array}{l}\text { Not sure/ } \\
\text { No } \\
\text { response }\end{array}$ & High & Medium & Low & Zero & $\begin{array}{l}\text { Not sure/ } \\
\text { No } \\
\text { response }\end{array}$ \\
\hline Automation & 1 & 6 & 0 & 0 & 1 & 4 & 5 & 2 & 2 & 2 \\
\hline Autonomy & 2 & 5 & 0 & 0 & 1 & 4 & 2 & 2 & 3 & 4 \\
\hline Bottom-up initiatives & 1 & 4 & 1 & 0 & 2 & 4 & 5 & 2 & 0 & 4 \\
\hline Career prospects & 4 & 1 & 2 & 0 & 1 & 4 & 8 & 2 & 0 & 1 \\
\hline Communication & 4 & 3 & 0 & 0 & 1 & 3 & 10 & 2 & 0 & 0 \\
\hline Compulsory & 1 & 4 & 2 & 0 & 1 & 1 & 5 & 5 & 1 & 3 \\
\hline Cost beneficial & 6 & 1 & 0 & 0 & 1 & 6 & 5 & 4 & 0 & 0 \\
\hline Critical mass & 0 & 5 & 0 & 0 & 3 & 0 & 3 & 5 & 2 & 5 \\
\hline Eliminates bureaucracy & 3 & 5 & 0 & 0 & 0 & 3 & 5 & 5 & 0 & 2 \\
\hline Empowerment & 4 & 3 & 1 & 0 & 0 & 1 & 8 & 3 & 1 & 2 \\
\hline External audits & 1 & 6 & 0 & 0 & 1 & 4 & 6 & 3 & 0 & 2 \\
\hline Feedback & 3 & 5 & 0 & 0 & 0 & 6 & 7 & 2 & 0 & 0 \\
\hline Job satisfaction & 3 & 3 & 0 & 0 & 2 & 8 & 7 & 0 & 0 & 0 \\
\hline Justifiable benefits & 2 & 5 & 0 & 0 & 1 & 3 & 10 & 2 & 0 & 0 \\
\hline $\begin{array}{l}\text { Knowledgeable team } \\
\text { leaders }\end{array}$ & 4 & 4 & 0 & 0 & 0 & 8 & 6 & 1 & 0 & 0 \\
\hline $\begin{array}{l}\text { Maintainable/easy } \\
\text { processes }\end{array}$ & 4 & 3 & 0 & 0 & 1 & 11 & 4 & 0 & 0 & 0 \\
\hline Meeting targets & 2 & 4 & 0 & 0 & 2 & 7 & 6 & 0 & 1 & 1 \\
\hline Phased introduction & 1 & 2 & 1 & 0 & 4 & 0 & 5 & 4 & 1 & 5 \\
\hline Process ownership & 2 & 5 & 0 & 1 & 0 & 2 & 8 & 3 & 0 & 2 \\
\hline Reduced admin & 1 & 3 & 2 & 0 & 2 & 1 & 9 & 3 & 0 & 2 \\
\hline Resources & 4 & 3 & 1 & 0 & 0 & 3 & 9 & 2 & 0 & 1 \\
\hline Reward schemes & 2 & 2 & 2 & 0 & 2 & 4 & 3 & 6 & 0 & 2 \\
\hline Saleability & 1 & 3 & 1 & 0 & 3 & 3 & 6 & 3 & 0 & 3 \\
\hline Shared best practices & 4 & 1 & 1 & 0 & 2 & 7 & 8 & 0 & 0 & 0 \\
\hline SPI forum & 1 & 3 & 0 & 1 & 3 & 3 & 4 & 3 & 2 & 3 \\
\hline Standardisation & 3 & 3 & 1 & 0 & 1 & 6 & 7 & 1 & 0 & 1 \\
\hline Taller hierarchy & 2 & 4 & 1 & 0 & 1 & 3 & 4 & 4 & 1 & 3 \\
\hline Task forces & 3 & 1 & 3 & 0 & 1 & 6 & 7 & 2 & 0 & 2 \\
\hline Top-down commitment & 5 & 1 & 1 & 0 & 1 & 8 & 5 & 1 & 0 & 1 \\
\hline Training & 2 & 6 & 0 & 0 & 0 & 5 & 10 & 0 & 0 & 0 \\
\hline Visible success & 5 & 2 & 0 & 0 & 1 & 5 & 9 & 1 & 0 & 0 \\
\hline
\end{tabular}

For the respondents from large companies, it was found that all of them perceived 'top-down commitment' as a 'high' value motivator. Other frequently cited 'high' perceived value motivators for respondents of large companies are: cost beneficial, rewards schemes, and shared best practices. We have found that 'process ownership' and 'SPI forum' also motivate the employees of large companies to support SPI programmes in their companies.

\section{LIMITATION}

There are some limitations that we considered worth mentioning. A disadvantage of the questionnaire survey method is that respondents are provided with a list of possible motivators and asked to select from that list. This tends to preempt the motivators investigated and to limit them to those reported in existing studies - respondents only focus on the motivators provided in the list. It is also possible that respondents may misinterpret the motivator provided in the questionnaire. However, we tried to address this issue by explaining the meaning of each motivator included in the questionnaire (Please see Appendix A for meanings of the motivators). Another issue is that the questionnaire surveys are usually based on self-reported data that reflects what people say happened, not necessarily what they actually did 
or experienced. Our results are limited to the respondents' knowledge, attitudes, and beliefs regarding the factors that motivate them to support SPI initiatives in their company. This situation can cause problems when practitioners' perceptions may be inaccurate or factors identified as important SPI motivators may not be important at all. However, like the researchers of many studies based on opinion data (e.g. (Baddoo and Hall, 2002; Beecham et al, 2003; Niazi et al, 2006)), we also have full confidence in our findings because we have collected data from practitioners working in quite diverse roles and directly involved in SPI activities within their companies. Sample size may be another issue as we collected data from only 23 practitioners from 8 Vietnamese companies. To gain a broader representation of Vietnamese practitioners' views on this topic, more practitioners and companies need to be included in a study. Our participants belonged to only one country, Vietnam, which is another limitation as the findings cannot be widely generalised to practitioners from other countries.

\section{SUMMARY AND CONCLUSION}

We report on our empirical study of SPI motivators. We analysed the experiences, opinions and views of practitioners in order to identify factors that motivate them for SPI initiatives. Five types of SPI motivators assessments (high, medium, low, zero, do not know) were identified that have led us to the notion of a 'perceived value' associated with each SPI motivator (from high value to no value). To have a perceived high value, we refer to a motivator that is common across all practitioners.

In order to describe the notion of perceived value within SPI motivators, we have used the following criterion:

- If the majority of respondents $(\geq 50 \%)$ thought that a motivator had high value then we treat that motivator as critical.

In order to answer RQ1, using above criterion, we have identified 6 'high' perceived value SPI motivators that are generally considered critical for successfully implementing SPI initiatives: cost beneficial, job satisfaction, knowledgeable team leaders, maintainable/easy processes, shared best practices, and top-down commitment. We have identified $10 \mathrm{SPI}$ motivators that have 'medium' perceived value: automation, communication, empowerment, external audits, feedback, justifiable benefits, process ownership, resources, training, and reduced admin.

In order to answer RQ2, our results also show the opinion of each individual practitioner group. Developers are highly motivated by: career prospects, communication, cost beneficial, empowerment, knowledgeable team leaders, maintainable/easy processes, resources, shared best practices, top-down commitment, and visible success; Managers are highly motivated by: job satisfaction, knowledgeable team leaders, maintainable/easy processes, meeting targets, shared best practices, and top-down commitment.

Analysis of the responses based on the company size of the respondents shows that the practitioners working for small-medium size companies are highly motivated to support SPI initiatives by factors such as cost beneficial, job satisfaction, knowledgeable team leaders, and maintainable/easy processes. The large companies' practitioners are more motivated to support SPI initiatives by factors such as career prospects, cost beneficial, eliminate bureaucracy, feedback, job satisfaction, maintainable/easy processes, rewards schemes, shared best practices, task force, topdown commitment, training, and visible success.

We have found that 4 'high' perceived value motivators common to all practitioner groups are: knowledgeable team leaders, maintainable/easy processes, shared best practices, top-down commitment. We have also found 4 'medium' perceived value motivators common to all practitioner are: feedback, justifiable benefits, process ownership, and training. We have also identified 3 'high' perceived value motivators common between practitioners of small-medium and large companies: cost beneficial, job satisfaction, and maintainable/easy processes. We suggest that focusing on these common motivators can help SPI practitioners to design and implement strategies for successful SPI programmes in their companies.

As part of a large project about the SPI motivation, we have been carrying out a research project to investigate the SPI motivators in the Asia-pacific region. This paper reports the findings of one part of our research project aimed at identifying the factors that are perceived by Vietnamese software practitioners as SPI motivators. To gain a broader representation of practitioners' views on this topic, we have also collected data from Malaysian practitioners. In our future work we will analyse the Malaysian practitioners views on SPI motivation. The results of this project are expected not only help software practitioners to understand the usage of SPI motivators in the Asia-pacific region but will also help them to compare SPI motivators identified in other regions (Baddoo and Hall, 2002; Khalil et al, 1997).

ACKNOWLEDGEMENT: We are thankful to the management of the following companies for making their staff available for this project: AZ, CT-IN, FPT, Goodland, NCS, Quantics, SDC, and VietSoftware. This research was carried out when the second author was working with National ICT, Australia (NICTA). 


\section{Motivators of Software Process Improvement: An Analysis of Vietnamese Practitioners' Views}

\section{REFERENCES}

Ashrafi, N. 2003. The impact of software process improvement on quality: in theory and practice, Information \& Management 40 (7). 677-690.

Baddoo, N. and Hall, T. 2002. Motivators of software process improvement: An analysis of practitioner's views, Journal of Systems and Software (62). 85-96.

Baddoo, N., Hall, T. and Wilson, D. 2000. Implementing a people focused SPI programme. 11th European Software Control And Metrics Conference and The Third SCOPE Conference on Software Product Quality, Munich.

Basili, V. R., McGarry, F. E., Pajerski, R. and Zelkowitz, M. V. 2002. Lessons learned from 25 years of process improvement: The rise and fall of the NASA software engineering laboratory. International Conference on Software Engineering, Orlando, Florida, USA. 69-79.

Beecham, S., Tracy, H. and Austen, R. 2003. Software Process Problems in Twelve Software Companies: An Empirical Analysis, Empirical software engineering 8 7-42.

Butler, K. 1995. The economics benefits of software process improvement, CrossTalk (July).

Butler, K. 1997. Process lessons learned while reaching Level 4, CrossTalk (May).

Chidamber, S. R. 2003. An Analysis of Vietnam's ICT and Software Services Sector, The Electronic Journal on Information Systems in Developing Countries. http://www.ejisdc.org, Last accessed 01 Nov 2005, 1-11.

Coolican, H. 1999. Research Methods and Statistics in Psychology. Hodder and Stoughton, London.

Davis, A. and Hickey, A. 2002. Requirements Researchers: Do We Practice What We Preach?, Requirements Engineering Journal 2002 (7). 107-111.

Fitzgerald, B. and O'Kane, T. 1999. A longitudinal study of software process improvement, IEEE Software (May/June). 37-45.

Florence, A. 2001. Lessons learned in attempting to achieve software CMM Level 4, CrossTalk (August). 29-30.

Fowler, P., Middlecoat, B. and Yo, S. 1999. Lessons learned collaborating on a process for SPI at Xerox. Technical report, CMU/SEI-99-TR-006, Software Engineering Institute, USA.

Goldenson, D. R. and Herbsleb, J. D. 1995. After the appraisal: A systematic survey of Process Improvement, Its benefits, And Factors That Influence Success. SEI, CMU/SEI-95-TR-009, Software Engineering Institute, USA.

Hall, T. and Wilson, D. 1997. Views of software quality: a field report, IEEE Proceedings on Software Engineering 144 (2). 111118.

Hall, T. and Wilson, D. 1998. Perceptions of software quality: a pilot study, Software quality journal (7). 67-75.

Herbsleb, J. D. and Goldenson, D. R. 1996. A systematic survey of CMM experience and results. 18th international conference on software engineering (ICSE-18). Berlin, Germany. 323-330.

ISO/IEC-15504. 1998. Information technology - Software process assessment. Technical report - Type 2.

Jiang, J., Klein, G., Hwang, H.-G., Huang, J. and Hung, S.-y. 2004. An exploration of the relationship between software development process maturity and project performance, Information \& Management (41). 279-288.

Kaltio, T. and Kinnula, A. 2000. Deploying the defined software process, Software Process - Improvement and Practice (5). 65-83. Kautz, K. and Nielsen, P. A. 2000. Implementing software process improvement: Two cases of technology transfer. Proceedings of the 33rd Hawaii Conference on System Sciences, Volume 7, Maui, USA. 1-10.

Khalil, O. E. M., Zawacki, R. A., Zawacki, P. A. and Selim, A. 1997. What Motivates Egyptian IS Managers and Personnel: Some Preliminary Results. SIGCPR 97. 187-196.

McDermid, J. and Bennet, K. 1999. Software Engineering research: A critical appraisal, IEE Proceedings on software engineering 146 (4). 179-186.

Moitra, D. 1998. Managing change for (SPI) initiatives: A practical experience-based approach, Software Process Improvement and Practice 4 (4). 199-207.

Niazi, M. 2006. Software Process Improvement: A Road to success. The 7th International Conference on Product Focused Software Process Improvement, LNCS. 395-401.

Niazi, M., Cox, K. and Verner, J. 2005a. An empirical study identifying high perceived value requirements engineering practices. Fourteenth International Conference on Information Systems Development (ISD’2005) Karlstad University, Sweden August 15-17. Niazi, M., Wilson, D. and Zowghi, D. 2004. Critical Barriers for SPI Implementation: An empirical study. IASTED International Conference on Software Engineering (SE 2004). Austria. 389-395.

Niazi, M., Wilson, D. and Zowghi, D. 2005b. A Maturity Model for the Implementation of Software Process Improvement: An empirical study, Journal of Systems and Software 74 (2). 155-172.

Niazi, M., Wilson, D. and Zowghi, D. 2006. Critical Success Factors for Software Process Improvement: An Empirical Study, Software Process Improvement and Practice Journal 11 (2). 193-211.

Paulk, M., Curtis, B., Chrissis, M. and Weber, C. 1993. Capability Maturity Model for software, Version 1.1. CMU/SEI-93-TR-24, Software Engineering Institute USA.

Pitterman, B. 2000. Telcordia Technologies: The journey to high maturity, IEEE Software (July/August). 89-96. 
Rainer, A. and Hall, T. 2002a. Key success factors for implementing software process improvement: a maturity-based analysis, Journal of Systems \& Software (62). 71-84.

Rainer, A. and Hall, T. 2002b. Key success factors for implementing software process improvement: a maturity-based analysis, Journal of Systems \& Software 62 (2). 71-84.

SEI. 2002. Capability Maturity Model® Integration (CMMISM), Version 1.1. SEI, CMU/SEI-2002-TR-029, Software Engineering Institute, USA.

SEI. 2004. Process Maturity Profile. Software Engineering Institute Carnegie Mellon University,

Sharp, H., Woodman, M., Hovenden, F. and Robinson, H. 1999. The role of 'culture' in successful software process improvement. 25th EUROMICRO Conference.

Trewin and D. 2002. Small Business in Australia: 2001. Australian Bureau of Statistics report 1321.0,

Warden, R. and Nicholson, I. 1995. IT quality initiatives at risk, Software Quality Management, 24, 24-27.

Wilson, D., Hall, T. and Baddoo, N. 2001. A framework for evaluation and prediction of SPI success, Systems and software (59). 135-142.

Yamamura, G. 1999. Software process satisfied employees, IEEE Software (September/October). 83-85.

\section{Appendix A: Definition of SPI Motivators (Baddoo and Hall, 2002)}

\begin{tabular}{|l|l|}
\hline Motivator & Definition \\
\hline Automation & Tools to eliminate paper work \\
\hline Autonomy & Enables practitioners to perform different roles \\
\hline Bottom-up initiatives & Lowe and middle management have input into the design and planning of SPI \\
\hline Career prospects & Improves career prospects \\
\hline Communication & Improved communication about SPI \\
\hline Compulsory & All SPI practices are made mandatory \\
\hline Cost beneficial & Improved cost/revenue ratio through SPI \\
\hline Critical mass & The presence of sufficient staff members who want to see SPI happen \\
\hline Eliminates bureaucracy & Eliminates spending time on bureaucratic processes \\
\hline Empowerment & Empower staff to take decisions on SPI \\
\hline External audits & Provision of some external body to maintain SPI practices \\
\hline Feedback & Feedback from stakeholders \\
\hline Job satisfaction & Practitioners get job satisfaction from producing good quality process \\
\hline Justifiable benefits & The ability to justify the long-term benefits of SPI \\
\hline Knowledgeable team leaders & Having team leaders who know about SPI \\
\hline Maintainable/easy processes & Processes that are easy to understand, follow and maintain \\
\hline Meeting targets & SPI practice help company to meet company's goals \\
\hline Phased introduction & SPI is introduced through small and incremental implementation \\
\hline Process ownership & Stakeholders own and therefore are able to change processes \\
\hline Resources & Sufficient time and resources allocated to SPI \\
\hline Reward schemes & Stakeholders are rewarded for SPI work \\
\hline Saleability & The perception that SPI will lead to more saleable job market skills \\
\hline Shared best practices & Best practice is shared in companies \\
\hline SPI forum & Creating a forum where SPI ideas can be discussed \\
\hline Standardisation & SPI makes practitioners work in a standardised way \\
\hline Taller hierarchy & Taller company hierarchies for more opportunity and promotion \\
\hline Task forces & Using task forces for SPI \\
\hline Top-down commitment & Senior management support for SPI \\
\hline Training & Training provided to practitioners in SPI practices \\
\hline Reduced admin & SPI leads to reduced administration \\
\hline Visible success & Evidence of the benefits of SPI \\
\hline
\end{tabular}

